\title{
HAMILTONIAN ENGINEERING FOR QUANTUM SYSTEMS
}

\author{
Sonia G Schirmer \\ Dept of Applied Math 63 Theoretical Physics, University of \\ Cambridge, Cambridge, CB3 0WA, United Kingdom
}

\begin{abstract}
We describe different strategies for using a semi-classical controller to engineer quantum Hamiltonians to solve control problems such as quantum state or process engineering and optimization of observables. Copyright (C) 2006 IFAC.
\end{abstract}

\section{INTRODUCTION}

Extending control to the quantum domain, i.e., to physical systems whose behavior is not governed by classical laws but dominated by quantum effects, has become an important area of research recently. It is also an essential prerequisite for the development of novel technologies such as quantum information processing, as well as new applications in quantum optics, quantum electronics, or quantum chemistry. Choreographing the behavior of interacting quantum particles is a rather difficult task in general, for a variety of reasons, including the destructive effects of uncontrollable interactions with the environment and measurement backaction, both of which lead to loss of coherence, for instance. Yet, many problems in quantum optics, quantum electronics, atomic physics, molecular chemistry, and quantum computing can be reduced to quantum state or process engineering, and optimization of observables, which can be solved using Hamiltonian engineering techniques. In this paper we outline and compare different strategies for accomplishing this in various quantum settings.

\section{CONTROL SYSTEM MODEL}

A control system must obviously consist of a system to be controlled and a controller. In quantum control the former is always quantum-mechanical. The latter can be either quantum or classical, and it may seem natural to choose another quantum system as the controller. Indeed, this is useful for some applications, for instance in quantum optics [Yanagisawa and Kimura (2003)]. The main challenge in general though, and the focus of this paper, is control at the interface between the quantum world and the classical world we experience, i.e., control of systems that obey the laws of quantum physics using semi-classical sensors and actuators that interact with the quantum system but accept classical input (such as different settings of the classical control switches of the laboratory equipment) and return classical information (See Fig. 1).

This model is applicable to many quantum control settings. For example, the quantum system could be an ensemble of molecules involved in a chemical reaction, subject to laser pulses produced by an actuator consisting of a laser source and pulse shaping equipment, and a detector that might consist of a mass spectrometer to identify the reaction products. It could be a solid-state system such as an ensemble of quantum dots representing qubits, with actuators and sensors consisting of control electrodes and single-electron transistors, respectively. It could be an ensemble of molecules with nuclear spins subject to actuators generating magnetic and radio-frequency fields, and sensors that detect the magnetization of the sample, etc.

\section{SYSTEM DYNAMICS}

In the setting defined above the state of the system can be represented either by a Hilbert space vector $|\Psi(t)\rangle$, or more generally, a density operator $\rho(t)$, i.e., a positive trace-one operator acting on the system's Hilbert space $\mathcal{H}$. Although the full Hilbert spaces of most quantum systems are hardly finite or separable - even the Hilbert space of the hydrogen atom is not separable if the full continuum of ionized states is included, we are usually only interested in controlling the dynamics of a finite-dimensional subspace of the system's full Hilbert space (e.g., a subset of bound states of an atom), and full control of an infinitedimensional system is generally not possible in 


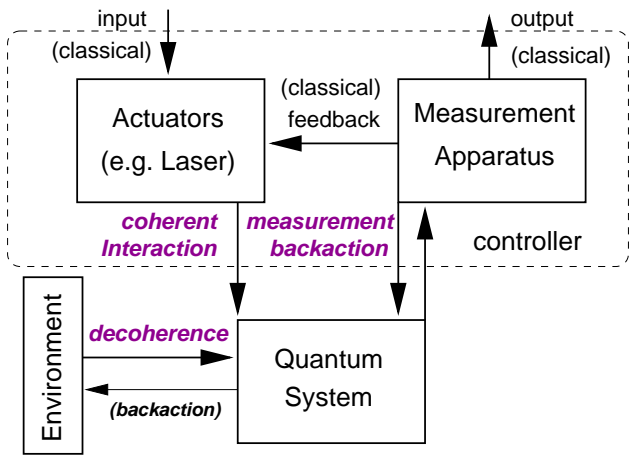

Fig. 1. Semi-classical Quantum Control Model

any case [see e.g. Tarn et al. (2000)]. We will therefore assume in this paper that the Hilbert spaces of interest are finite-dimensional, and thus all operators have matrix representations, etc.

Neglecting decoherence and the effect of measurements for a moment, the evolution of the quantum system to be controlled is governed by the Schrodinger equation

$$
\frac{d}{d t}|\Psi(t)\rangle=-\frac{i}{\hbar} H[\mathbf{f}(t)]|\Psi(t)\rangle,
$$

or the (equivalent) quantum Liouville equation

$$
\frac{d}{d t} \rho(t)=-\frac{i}{\hbar}[H[\mathbf{f}(t)], \rho(t)],
$$

where $[A, B]=A B-B A$ is the commutator. $\hbar=h / 2 \pi$, where $h$ is the Planck constant, but we will often choose units such that $\hbar=1$. Thus, the dynamics is determined by the Hamiltonian $H[\mathbf{f}(t)]$, which is an operator acting on the system's Hilbert space $\mathcal{H}$, and depends on a set of (classical) control fields $\mathbf{f}(t)=\left(f_{1}(t), \ldots, f_{M}(t)\right)$ produced by the actuators.

The main difference between Eqs (1) and (2) is that the former applies only to pure-state systems, while the latter applies equally to pure and mixedstate systems (quantum ensembles). Moreover, unlike the Schrodinger equation, the quantum Liouville equation can be generalized for systems subject to decoherence or weak measurements by adding (non-Hamiltonian) superoperators [operators acting on Hilbert space operators such as $\rho(t)$ ] to account for the contributions of measurements and dissipation to the dynamics of the system

$$
\dot{\rho}(t)=\mathcal{L}_{H}[\rho(t)]+\mathcal{L}_{M}[\rho(t)]+\mathcal{L}_{D}[\rho(t)],
$$

where $\mathcal{L}_{H}[\rho(t)]=-\frac{i}{\hbar}[H[\mathbf{f}(t)], \rho(t)]$. The main difference between $\mathcal{L}_{M}$ and $\mathcal{L}_{D}$ is conceptual: the former depends on the measurements performed, i.e., the configuration of the sensors, which we can control, the latter on usually uncontrollable interactions of the system with the environment.

Due to the physically prescribed evolution equations for quantum systems, quantum control - at least in the classical controller model describedis fundamentally non-linear, even in the case of open-loop control. In the special case when the system's interaction with both sensors and the environment is negligible (which is obviously only possible for open-loop control), the system's dynamics is completely determined by a Hamiltonian operator $H[\mathbf{f}(t)]$, and the main task of quantum control is to find effective ways to engineer this Hamiltonian to achieve a desired objective. For simplicity we will restrict our attention here mostly to Hamiltonian engineering in this case, although many of the techniques are still useful in the more there general case when the system is subject to measurements and/or uncontrollable interactions with the environment [Eq. (3)].

\section{QUANTUM CONTROL OBJECTIVES}

Although the objectives vary depending on the application, most problems in quantum control can be reduced to quantum process engineering, quantum state engineering or optimization of observables. The first, quantum process engineering, involves finding a Hamiltonian $H[\mathbf{f}(t)]$ such that

$$
\exp _{+}\left[-i \int_{t_{0}}^{t_{F}} H[\mathbf{f}(t)] d t\right]=U
$$

where $U$ is a desired target process, and the subscript + indicates that the exponential must be interpreted as a time-ordered exponential with positive time-ordering due to the time-dependent nature of the Hamiltonian. This problem is of particular interest in quantum computing, where the target processes are quantum logic gates. In general, not every process can be implemented for a given system, and the set of reachable processes is determined by the dynamical Lie group associated with the set of Hamiltonians $\{H[\mathbf{f}(t)]: \mathbf{f}(t) \in \mathcal{A}\}$, where $\mathcal{A}$ is the set of admissible controls that can be produced by the actuators and satisfy physical constraints, e.g., on the field strength, etc. For example, for a Hamiltonian system a necessary (minimum) requirement is that $U$ be a unitary operator (acting on the system's Hilbert space), and other constraints may restrict the set of reachable operators further. However, if the target process is reachable then there are usually many Hamiltonians, which will give rise to the same process.

The second problem, quantum state engineering, requires finding a Hamiltonian $H[\mathbf{f}(t)]$ such that (a) $\left|\Psi\left(t_{F}\right)\right\rangle=\left|\Psi_{1}\right\rangle,\left|\Psi\left(t_{0}\right)\right\rangle=\left|\Psi_{0}\right\rangle$, and $|\Psi(t)\rangle$ satisfies the dynamical equation (1), or (b) $\rho\left(t_{F}\right)=$ $\rho_{1}, \rho\left(t_{0}\right)=\rho_{0}$, and $\rho(t)$ satisfies the dynamical equation (2) [or (3) in the general case], where $\rho_{0}$ $\left[\left|\Psi_{0}\right\rangle\right]$ and $\rho_{1}\left[\left|\Psi_{0}\right\rangle\right]$ represent the initial and target state, respectively. 
The final problem, optimization of observables, requires finding a Hamiltonian such that the expectation value (or ensemble average) of an observable $A, \operatorname{Tr}\left[A \rho\left(t_{F}\right)\right]$, assumes a maximum or minimum at a certain time $t_{F}$, given that the state $\rho(t)$ evolves according to Eq. (2) and satisfies an initial condition $\rho\left(t_{0}\right)=\rho_{0}$. Problems of this type arise frequently in atomic and molecular physics and chemistry, where the observables of interest can range from the position or momentum of a particle, to the dipole moment or the vibrational energy of a molecular bond, etc., but they are also relevant in quantum computing where we wish to maximize the gate fidelity or the projection of the system onto subspaces that are robust with regard to decoherence, etc.

While the second problem may appear much simpler than the first, it can be shown that for a generic quantum ensemble, i.e., a quantum ensemble described by a density operator $\rho$ with a maximum number of distinct eigenvalues, the problems of quantum state and process engineering are essentially equivalent, up to a usually unobservable (and hence insignificant) global phase factor. Similarly, the third problem is equivalent to quantum process engineering if the initial state of the system is a generic quantum ensemble and the target observable is represented by an operator $A$ with distinct eigenvalues (occurring with multiplicity 1). It should be noted, however, that this equivalence does not hold for pure-state systems, which are always represented by density operators of rank 1 , and for which process engineering is in general a much harder problem than the others. [See e.g. Schirmer et al. (2002a)]

\section{HAMILTONIAN ENGINEERING}

Having shown how many problems in quantum control can be reduced to Hamiltonian engineering problems, we shall now consider various strategies for finding and implementing control Hamiltonians for quantum systems, and their advantages and drawbacks. So far, we have not made any assumptions about the structure of the Hamiltonian $H[\mathbf{f}(t)]$ or the nature of the control fields $\mathbf{f}(t)$ applied, both of which depend on the specific physical systems considered. While we will try to avoid being too specific some additional assumptions are necessary.

We can always partition $H[\mathbf{f}(t)]$ into a system part $H_{S}$, which describes the system's intrinsic dynamics and is independent of the controller, and a control part $H_{C}[\mathbf{f}(t)]$. Although $H_{C}[\mathbf{f}(t)]$ can depend on the control functions $f_{m}(t)$ in a nonlinear fashion, in many situations assuming a linear dependence on the field components $f_{m}(t)$

$$
H_{C}[\mathbf{f}(t)]=\sum_{m=1}^{M} f_{m}(t) H_{m},
$$

is a reasonably good approximation. Furthermore, while in some applications such as solid-state architectures with multiple control electrodes, there are naturally multiple independent fields, in many applications there is only a single effective control field such as the electromagnetic field induced by a laser pulse, a maser or radio-frequency field, for instance, and thus the control Hamiltonian simplifies further $H_{C}[\mathbf{f}(t)]=f(t) H_{1}$.

In the control-linear case (5), a necessary and sufficient condition for being able to engineer any (unitary) process up to a global phase factor is that the Lie algebra generated by $i H_{S}$ and $i H_{m}$, $m=1, \ldots, M$ is either $u(N)$ or $s u(N)$, where $N$ is the dimension of the relevant Hilbert space $\mathcal{H}$ [Albertini and D'Alessandro (2001); Schirmer et al. (2002b)]. As outlined in the previous section, this is also a necessary and sufficient condition for quantum state or observable controllability, at least for generic quantum ensembles and observables. Most quantum systems can be shown to be controllable, but constructive control can be challenging.

\subsection{Geometric Control Techniques}

Given a set of (independent) control Hamiltonians $H_{m}, m=1, \ldots, M$, which is complete in that the $i H_{m}$ generate the entire Lie algebra, the simplest general strategy we can pursue is to expand the (unitary) target process $U$ into a product of elementary (complex) rotations $\exp \left(i c H_{m}\right)$ in a cyclic, iterating pattern

$$
U=\prod_{k=1}^{K}\left[\prod_{m=1}^{M} \exp \left(i c_{k m} H_{m}\right)\right]
$$

and determine the constants $c_{k m}$ in the expansion using Lie group decomposition methods, from which we can derive suitable values for the field strengths $f_{m k}$ and control pulse lengths $t_{m k}$ via the relation $c_{m k}=\int_{0}^{t_{m k}} f_{m k}(t) d t$, which reduces to $c_{m k}=f_{m k} t_{m k}$ for piecewise constant fields.

If the $H_{m}$ are orthogonal, i.e., $\operatorname{Tr}\left[H_{m} H_{n}\right]=$ const. $\delta_{m n}$, there are well-developed geometric techniques to solve this type of problem. Explicit solutions have been developed especially for many problems involving spin $-1 / 2$ particles, which are of interest in nuclear magnetic resonance (NMR) applications [e.g. D'Alessandro (2000)], and even constructive algorithms for the generation of arbitrary unitary operators on $n$-qubits via $S U\left(2^{n}\right)$ decompositions have been proposed recently [Sa Earp and Pachos (2005)]. For non-qubit systems the problem is harder but some explicit decomposition algorithms for simple $N$-level systems 
have also been proposed [e.g. Ramakrishna et al. (2000b)].

In practice, however, most physical systems are subject to internal system dynamics in addition to the control-induced dynamics. This leads to a drift term $H_{S}$, which is usually non-trivial and cannot be turned off. In principle, we can include this term in the expansion (6) by replacing the control Hamiltonians $H_{m}$ by $H_{S}+H_{m}$, for example. However, even if the $H_{m}$ are orthogonal, the new effective Hamiltonians in the expansion are usually not, which significantly complicates the problem. Moreover, even if we find a decomposition for given a problem, it may not be physically realizable as the $c_{k m}$ could be negative. Since the control coefficient corresponding to $H_{S}$ is fixed $\left(f_{S}=\right.$ $1)$, implementing such a rotation would require letting the system evolve for negative times, which is usually impossible (except possibly if the evolution of the system is periodic). Decomposition techniques for non-orthogonal Hamiltonians that take these constraints into account exist for $S U(2)$ [Ramakrishna et al. (2000a)] but even in this simple case the resulting pulse sequences are much more complicated and for higher-dimensional systems even the minimum number of pulses necessary to generate a given unitary transformation given non-orthogonal Hamiltonians is generally not known.

The usual way to circumvent the problem of drift is by transforming to a rotating frame $(\mathrm{RF})$ given by $e^{-i t E_{n}}|n\rangle$, where $\{|n\rangle: n=1, \ldots, N=\operatorname{dim} \mathcal{H}\}$ is a basis of $\mathcal{H}$ consisting of eigenstates $|n\rangle$ of $H_{S}$ with eigenvalues (energies) $E_{n}, H_{S}|n\rangle=E_{n}|n\rangle$. Setting $U_{S}(t)=\exp \left(-i t H_{S}\right)$ the dynamics in the rotating frame is governed by the new (interaction picture) Hamiltonian

$$
H_{C}^{\prime}[\mathbf{f}(t)]=U_{S}(t)^{\dagger} H_{C}[\mathbf{f}(t)] U_{S}(t) .
$$

Thus we have transformed away the drift term but the previously time-independent Hamiltonians $H_{m}$ in the control-linear approximation are now time-dependent

$$
H_{m}^{\prime}[\mathbf{f}(t)]=U_{S}(t)^{\dagger} H_{m} U_{S}(t) .
$$

Thus further approximations such as spectral decomposition of the control fields into a small number of distinct frequency components are needed, e.g.,

$$
f(t)=\sum_{n, n^{\prime}>n} A_{n n^{\prime}}(t) \cos \left(\omega_{n n^{\prime}} t+\phi_{n n^{\prime}}\right),
$$

where $\omega_{n n^{\prime}}=\left(E_{n^{\prime}}-E_{n}\right) / \hbar$ is the transition frequency between states $\left|n^{\prime}\right\rangle$ and $|n\rangle, A_{n n^{\prime}}(t)$ are "amplitude functions" and $\phi_{n n^{\prime}}$ constant phases. If the system is strongly regular, $\omega_{n n^{\prime}} \neq \omega_{m m^{\prime}}$ unless $\left(n, n^{\prime}\right)=\left(m, m^{\prime}\right)$, and the transition frequencies $\omega_{n n^{\prime}} / \hbar$ are well separated, then choosing control fields of the form (9) with slowly varying am- plitude functions $A_{n n^{\prime}}(t)$ compared to $2 \pi / \omega_{n n^{\prime}}$, enables us to address individual transitions via frequency selective pulses, and assume the following decomposition of the control Hamiltonian

$$
H_{C}^{\prime}=\sum_{n, n^{\prime}>n} f_{n n^{\prime}}(t) U_{S}(t)^{\dagger} H_{n n^{\prime}} U_{S}(t)
$$

where $f_{n n^{\prime}}(t)=A_{n n^{\prime}}(t) \cos \left(\omega_{n n^{\prime}} t+\phi_{n n^{\prime}}\right)$. We can further decompose the fields into rotating and counter-rotating terms since $2 \cos \left(\omega_{n n^{\prime}} t+\phi\right)=$ $e^{+i\left(\omega_{n n^{\prime}} t+\phi\right)}+e^{-i\left(\omega_{n n^{\prime}} t+\phi\right)}$. Noting that $U_{S}(t)$ is diagonal with respect to the eigenbasis $|n\rangle$ with elements $e^{-i t E_{n}}$, and making the rotating wave approximation (RWA), i.e., assuming that the pulses are sufficiently long that the contribution of the counter-rotating terms averages to zero, we obtain the simplified RWA control Hamiltonian

$$
H_{C}^{R W A}=\sum_{n, n^{\prime}>n} \Omega_{n n^{\prime}}(t) H_{n n^{\prime}}\left(\phi_{n n^{\prime}}\right)
$$

where $\Omega_{n n^{\prime}}=A_{n n^{\prime}}(t) d_{n n^{\prime}} / 2 \hbar$, and $H_{n n^{\prime}}\left(\phi_{n n^{\prime}}\right)=$ $x_{n n^{\prime}} \cos \phi_{n n^{\prime}}+y_{n n^{\prime}} \sin \phi_{n n^{\prime}}, d_{n n^{\prime}}$ being the transition dipole moment and $x_{n n^{\prime}}=|n\rangle\left\langle n^{\prime}|+| n^{\prime}\right\rangle\langle n|$. $y_{n n^{\prime}}=i\left(|n\rangle\left\langle n^{\prime}|-| n^{\prime}\right\rangle\langle n|\right)$. Thus, the evolution of the system is now governed by a control Hamiltonian that is drift-free with time-independent components $H_{n n^{\prime}}$, as desired.

The RF and RWA are ubiquitous in physics, and the RWA control Hamiltonian is the starting point for the design of control schemes using geometric techniques in many applications. As the derivation showed, however, it involves several simplifying assumptions such as strong regularity of the system, control dynamics much slower than the intrinsic dynamics of the system (control pulses must be much longer than the oscillation periods $2 \pi / \omega_{n n^{\prime}}$ for (9) and RWA to make sense), and the negligibility of off-resonant excitation. In reality, there are often further complications. E.g., for coupled spin systems one typically transforms into a multiply-rotating frame which leaves a residual drift term, which is usually neglected by assuming that the control pulses are sufficiently strong (hard) so that the influence of the drift term is negligible and the Hamiltonians can be assumed to be effectively orthogonal for the decomposition. Obviously, these assumptions are not always valid. [For an analysis of the validity of geometric control schemes for atomic and molecular systems see e.g. Schirmer et al. (2002a).]

\subsection{Optimal Strategies for Fast Control}

If we wish to achieve control on time-scales comparable to the intrinsic dynamics of the system, or wish to control systems with too many or insufficiently distinct transition frequencies, as is the case for complex molecular systems in chemistry for example, then a different approach is 
required. A promising alternative is to formulate the control problem in terms of optimization of an objective functional such as the distance from a target state or target process, the expectation value of an observable or the gate fidelity, subject to the constraint that the dynamical evolution equations (1), (2) or (3) be satisfied.

A particularly flexible approach is to formulate the control problem as a variational problem by defining a functional $\mathcal{J}=\mathcal{A}-\mathcal{D}-\mathcal{C}$, which incorporates the objective $\mathcal{A}$, the dynamical constraints $\mathcal{D}$ and the costs $\mathcal{C}$ and depends on variational trial functions, and to use variational calculus to find necessary and sufficient conditions for an extremum. For instance, a very popular choice in physical chemistry involves setting $\mathcal{A}=$ $\operatorname{Tr}\left[A \rho\left(t_{F}\right)\right]$, where $A$ is a (Hermitian) operator representing the objective (e.g., $A$ could be the projector $|\Psi\rangle\langle\Psi|$ onto a target state $|\Psi\rangle$ ), with a dynamical constraint functional given by

$$
\mathcal{D}=\int_{t_{0}}^{t_{F}} \operatorname{Tr}\left[A_{v}(t)\left(\dot{\rho}_{v}+\mathcal{L}\left[\rho_{v}(t)\right]\right)\right] d t
$$

where $\mathcal{L}\left[\rho_{v}(t)\right]=\mathcal{L}_{H}\left[\rho_{v}(t)\right]+\mathcal{L}_{M}\left[\rho_{v}(t)\right]+\mathcal{L}_{D}\left[\rho_{v}(t)\right]$ and $\rho_{v}(t)$ and $A_{v}(t)$ are variational trial functions for the state (density operator) and observable, respectively, and a cost term related to the control field energies

$$
\mathcal{C}=\sum_{m=1}^{M} \frac{\lambda_{m}}{2 \hbar} \int_{t_{0}}^{t_{F}}\left|f_{m}(t)\right|^{2}
$$

where $\lambda_{m}$ are penalty weights. Setting the independent variations of $\mathcal{J}$ with regard to $A_{v}, \rho_{v}$ and $f_{m}$ to 0 , a necessary condition for $\mathcal{J}$ to have an extremum, then leads to a set of coupled differential equations with mixed boundary conditions, the Euler-Langrange equations, which can be solved numerically to obtain a solution for the control fields $f_{m}(t)$ and the corresponding trajectories for the state $\rho(t)$ and the observable $A(t)$.

The key advantages of this approach are that we can deal with complex (and even non-linear) control Hamiltonians (no RWA or other approximations required), and are in fact not limited to Hamiltonian systems at all, and a wide range of costs or trade-offs can be taken into account. A drawback is that the Euler-Lagrange equations are almost always nontrivial and can only be solved using numerical techniques. However, efficient algorithms with good convergence properties exist for a large class of problems [see e.g. Maday and Turinici (2003)], and promising results have been obtained for various applications, especially laser control of molecular systems using ultrafast (sub-picosecond) pulses, a regime well outside the realm of applicability for frequency-selective geometric control schemes.

\subsection{Robust Control through Adiabatic Passage}

On the opposite end of the spectrum are adiabatic techniques. Rather than applying control fields to induce (fast) transitions between various states of the system by absorption or emission of field quanta (usually photons), adiabatic techniques rely on (slow) continuous deformation of the energy surfaces by strong, slowly varying control fields, and adiabatic following of the system's state, making use of the eigenstate decomposition of the Hamiltonian

$$
H[\mathbf{f}(t)]=\sum_{n=1}^{N} \epsilon_{n}(t)\left|\Psi_{n}(t)\right\rangle\left\langle\Psi_{n}(t)\right| .
$$

For a Hamiltonian with a control-induced timedependence the eigenvalues $\epsilon_{n}(t)$ and corresponding eigenstates $\left|\Psi_{n}(t)\right\rangle$ vary in time. The idea of adiabatic passage is that if we start in a particular initial state $\left|\Psi_{0}\right\rangle$, which will usually be an eigenstate of the system's intrinsic Hamiltonian $H_{S}$, and then slowly switch on and vary suitable control fields $\mathbf{f}(t)$, then rather than inducing spontaneous transitions to other states, the state $|\Psi(t)\rangle$ is going to remain an eigenstate of the Hamiltonian and follow the path determined by the control fields via Eq. (14). Adiabatic passage is the basis for many control schemes in atomic physics [Shore (1990)], most notably STIRAP.

The simplest example is population transfer in a three-level $\Lambda$-system (see Fig. 2) simultaneously driven by two fields $f_{m}(t)=A_{m}(t) \cos \left(\omega_{m} t\right)$, $m=1,2$, that resonantly exite the $1 \rightarrow 2$ and $2 \rightarrow 3$ transitions, respectively. In this case the RWA Hamiltonian (11) simplifies to

$$
H_{C}^{R W A}=-\left[\begin{array}{ccc}
0 & \Omega_{1}(t) & 0 \\
\Omega_{1}(t) & 0 & \Omega_{2}(t) \\
0 & \Omega_{2}(t) & 0
\end{array}\right]
$$

with $\Omega_{m}=d_{m, m+1} A_{m} / 2 \hbar$ for $m=1$, 2. Setting $\Omega=\sqrt{\Omega_{1}^{2}+\Omega_{2}^{2}}$ and $\theta(t)=\arctan \left[\Omega_{1}(t) / \Omega_{2}(t)\right]$, it is easy to verify that the eigenstates of this Hamiltonian are $\left|\Psi_{ \pm}(t)\right\rangle=\Omega_{1}|1\rangle \pm \Omega|2\rangle+\Omega_{2}|3\rangle$ for $\lambda_{ \pm}= \pm \Omega$, respectively, and $\left|\Psi_{0}(t)\right\rangle=\cos \theta(t)|1\rangle-$ $\sin \theta(t)|3\rangle$ for $\lambda_{0}=0$. Thus, if the system is initially in state $|1\rangle$, then applying control fields such that $\Omega_{1}(t) / \Omega_{2}(t)$ changes (sufficiently slowly) from 0 at $t_{0}$ to $\infty$ at $t_{F}$, results in adiabatic passage of the system from state $|1\rangle=\left|\Psi_{0}(0)\right\rangle$ to $|3\rangle=\left|\Psi_{0}\left(t_{F}\right)\right\rangle$ as $\theta(t)$ goes from 0 to $\pi / 2$. STIRAP is based on the realization that we can achieve this simply by applying two overlapping Gaussian pulses in a counter-intuitive sequence, i.e, so that $\Omega_{2}(t)$ starts and ends before $\Omega_{1}(t)$ as shown in Fig. 2. Since the upper level $|2\rangle$ is decoupled, i.e., not populated during this process, the transfer is robust against decay from the excited state, which is often a major limiting factor in the control of 


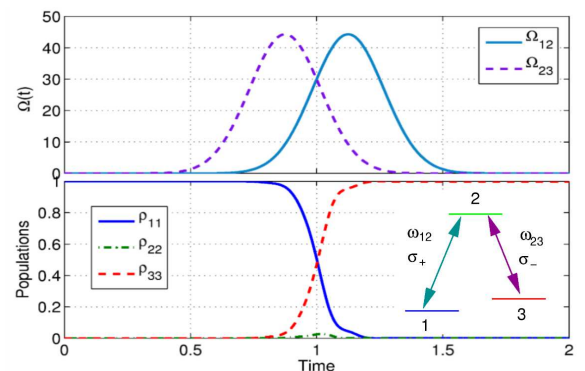

Fig. 2. STIRAP pulse sequence (top) for robust population transfer from state $|1\rangle$ to state $|3\rangle$ (bottom) for three-level $\Lambda$ system (inset).

atomic or molecular systems, although it must be noted that the scheme is sensitive with regard to other forms of decoherence etc.

\subsection{Learning Control via Closed-Loop Experiments}

A potential problem with all of the techniques described so far is their reliance on a model of the system. Unfortunately, such models are not always available, especially for complex systems. While improved techniques for quantum control system identification will hopefully eventually allow us to overcome this problem, an alternative which has already been successfully demonstrated in the laboratory [see e.g. Pearson et al. (2001)] are experimental closed-loop learning techniques [Judson and Rabitz (1992)], which must not be confused with real-time quantum feedback control [Wiseman (1994)]. While both schemes rely on (classical) information gained from measurements, the former involve repeated experiments on many copies of the system, while the latter rely on continuous observation of the same system via weak measurements (e.g. homodyne detection or passive photodetectors).

Closed-loop learning techniques essentially adaptive, using feedback to guide an evolutionary process. There are many variations but the basic strategy is simple. We define an objective functional, usually called fitness function, and select initial set (population) of control fields. Each of these is then applied to an identical copy of the system, and the observable measured to determine the fitness of the field. Then a new generation of control fields is computed from a subset of (mostly) well-performing fields using a set of predefined rules for mutations and crossovers, and the experiments are repeated with the new generation of fields until we have arrived at a population of fields with a sufficiently high fitness.

Although there are some disadvantages to this approach (requirement of many identical copies of the system or the ability to efficiently re-initialize the system in the same state after each experiment, need for high experimental duty cycles due to slow convergence, etc.), closed-loop learning techniques have proved useful in the laboratory for a wide range of systems, and incorporating feedback from such closed-loop learning experiments in some form is likely to be essential for quantum control to succeed in the laboratory. A particularly promising avenue may be adaptive system identification strategies based on feedback from closed-loop experiments.

\section{ACKNOWLEDGEMENTS}

This work was supported by the Cambridge-MIT Institute's Quantum Technology Project.

\section{REFERENCES}

F. Albertini, D. D'Alessandro. Notions of controllability for quantum-mechanical systems, 2001.

D. D'Alessandro. Algorithms for quantum control based on decompositions of Lie groups. In Proceedings of the 39th IEEE Conference on Decision and Control, pages 1074-1075, New York, 2000. IEEE. ISBN 0-7803-6638-7.

R. S. Judson, H. Rabitz. Teaching lasers to control molecules. Phys. Rev. Lett., 68:1500, 1992.

Y. Maday, G. Turinici. New formulations of monotonically convergent quantum control algorithms. J. Chem. Phys., 118(18):8191, 2003.

B. J. Pearson et al., Coherent control using adaptive learning algorithms. Phys. Rev. A, 63: 063412, 2001.

V. Ramakrishna et al., Quantum control by decompositions of $\mathrm{SU}(2)$. Phys. Rev. A, 62: 053409, 2000a.

V. Ramakrishna et al., Explicit generation of unitary transformations in a single atom or molecule. Phys. Rev. A, 61:032106, 2000b.

H. A. Sa Earp, J. K. Pachos. A constructive algorithm for the cartan decomposition of $S U\left(2^{n}\right)$. J. Math. Phys., 46:1, 2005.

S. G. Schirmer et al., Constructive control of quantum systems using factorization of unitary operators. J. Phys. A, 35:8315-8339, 2002a.

S. G. Schirmer, J. V. Leahy, A. I. Solomon. Degrees of controllability for quantum systems and applications to atomic systems. J. Phys. A, 35:4125, 2002b.

B. W. Shore. Theory of coherent atomic excitation. John Wiley \& Sons, New York, 1990.

T. J. Tarn, J. W. Clark, D. G. Lucarelli. Controllability of quantum-mechanical systems with continuous spectra. In Proceedings of the 39th IEEE Conference on Decision and Control, pages 2803-2809, New York, 2000. IEEE. ISBN 0-7803-6638-7.

H. M. Wiseman. Quantum theory of continuous feedback. Phys. Rev. A, 49:2133, 1994.

M. Yanagisawa and H. Kimura. Transfer function approach to quantum control. IEEE Trans. Autom. Control, 48:2107 and 2121, 2003. 